Mathematical Modelling and Analysis

Volume 16 Number 3, September 2011, 498-508

Doi:10.3846/13926292.2011.603167

(C) Vilnius Gediminas Technical University, 2011
Publisher: Taylor\&Francis and VGTU

www.tandfonline.com/loi/tmma20

Online ISSN: 1648-3510

Print ISSN: 1392-6292

\title{
Weak Solutions of Boundary Value Problems in Nontube Domains for Fourth-Order Equations of Composite Type
}

\section{Victor Korzyuk and Olga Kovnatskaya}

\author{
Belarusian State University \\ 4, Nezavisimosti av., Minsk 220030, Belarus \\ E-mail(corresp.): Korzyuk@bsu.by \\ E-mail: Kovnatskaya@bsu. by
}

Received August 30, 2010; revised July 3, 2011; published online August 1, 2011

\begin{abstract}
In the paper existence and uniqueness of weak solutions of boundary value problems in nontube domains for fourth-order equations of composite type are proved by methods of functional analysis.
\end{abstract}

Keywords: equation of composite type, weak solution, methods of functional analysis, partial differential equation of the fourth order.

AMS Subject Classification: 35M99; 35D30; 35G15.

\section{Introduction}

In the paper the existence and uniqueness of weak solution of boundary value problem in nontube domain for fourth-order equation of composite type are proved by methods of functional analysis. The main part of the operator is a composition of the wave operator and the operator of Laplace type. The analysis of such equations is caused by the need to develop further the fundamental theory of well-posed problems for linear partial differential equations, since such theory for the second-order equations of hyperbolic and elliptic types has been already developed. The interest to equations of composite type from a practical point of view is caused by a wide class of applications of in many fields of scientific knowledge and industry.

Boundary value problems for equations of composite type

$$
\frac{\partial}{\partial x} \Delta u=0, \quad \frac{\partial^{2}}{\partial x \partial y} \Delta u=0
$$

where $\Delta$ is Laplace operator and $\Delta=\frac{\partial^{2}}{\partial x^{2}}+\frac{\partial^{2}}{\partial y^{2}}, u=u(x, y)$ were considered by Hadamard $[12,13]$. It is possible to point to a number of papers $[1,4,5$, $6,7,8,9,10,11,18,19,24,25]$ where equation (1.1) and the other composite 
equations are investigated. We don't know any papers devoted to the equation we consider in this paper.

The purpose of this paper is to extend the theorems of existence and uniqueness of weak solutions of boundary value problems for the fourth-order equations of composite type in tube domains to nontube domains. A tube domain is a domain with the following property: the generator of the surface being part of the boundary of the domain and called lateral is parallel to the axis of $x_{0}$. We call nontube a domain which doesn't have this property. In the case of tube domains this problem was considered in the paper [20], the proof of existence and uniqueness of weak solutions of other problems in tube domains for this equation can be found in the papers [15, 20]. The papers [14] and [21] are devoted to proving existence and uniqueness of strong solutions of boundary value problems in tube domains for fourth-order equation of composite type. The equation of composite type of more common form is considered in the paper $[22]$.

\section{Statement of a Problem}

In a domain $Q$ of $(n+1)$-dimensional Euclidean space $\mathbb{R}^{n+1}$ of independent variables $\boldsymbol{x}=\left(x_{0}, \ldots, x_{n}\right)$ with respect to function $u: \mathbb{R}^{n+1} \supset Q \ni \boldsymbol{x} \rightarrow u(\boldsymbol{x}) \in$ $\mathbb{R}$ we consider a linear partial differential fourth-order equation of composite type

$$
\mathcal{L} u \equiv \frac{\partial^{4} u}{\partial x_{0}^{4}}+\left(b^{2}-a^{2}\right) \frac{\partial^{2}}{\partial x_{0}^{2}} \Delta u-a^{2} b^{2} \Delta^{2} u+A^{(2)} u=f(\boldsymbol{x}),
$$

where $a, b \in \mathbb{R}$ are given numbers satisfying the relation $a^{2}>b^{2}, \Delta=\sum_{i=1}^{n} \frac{\partial^{2}}{\partial x_{i}^{2}}$ is the Laplace operator with respect to the variables $\boldsymbol{x}^{\prime}=\left(x_{1}, \ldots, x_{n}\right), A^{(2)}=$ $\sum_{|\boldsymbol{\alpha}| \leqslant 2} a^{(\boldsymbol{\alpha})}(\boldsymbol{x}) \mathbf{D}^{\boldsymbol{\alpha}}, \mathbf{D}^{\boldsymbol{\alpha}}=\frac{\partial^{|\boldsymbol{\alpha}|}}{\partial x_{0}^{\alpha} \ldots \partial x_{n}^{\alpha_{n}}}, \boldsymbol{\alpha}=\left(\alpha_{0}, \ldots, \alpha_{n}\right), \alpha_{i}, i=0, \ldots, n$, are nonnegative integers, $|\boldsymbol{\alpha}|=\alpha_{0}+\ldots+\alpha_{n}$.

We denote by $C^{l}(\bar{Q})$ a set of continuously differentiable functions up to the order $l$ in the closure $\bar{Q}$ of the domain $Q$, where $l$ is nonnegative integer. In equation $(2.1) a^{(\boldsymbol{\alpha})}(\boldsymbol{x})$ are given functions and $a^{(\boldsymbol{\alpha})}(\boldsymbol{x}) \in C^{2}(\bar{Q})$.

Boundary $\partial Q$ of the domain $Q$ consists of three parts: $S_{1}=\{\boldsymbol{x} \in \partial Q$ $\left.\nu_{0}=-1\right\}, S_{2}=\left\{\left.\boldsymbol{x} \in \partial Q\left|\nu_{0}^{2}-a^{2}\right| \boldsymbol{\nu}^{\prime}\right|^{2} \leqslant-\delta_{1}\right\}$ and $S_{3}=\left\{\boldsymbol{x} \in \partial Q \mid \nu_{0}=1\right\}$, $\partial Q=S_{1} \cup S_{2} \cup S_{3}$, where $\boldsymbol{\nu}(\boldsymbol{x})=\left(\nu_{0}(\boldsymbol{x}), \ldots, \nu_{n}(\boldsymbol{x})\right)$ is the outward with respect to the domain $Q$ unit normal on the hypersurface $\partial Q$ at a point $\boldsymbol{x} \in \partial Q$, $\left|\boldsymbol{\nu}^{\prime}\right|^{2}=\nu_{1}^{2}+\ldots+\nu_{n}^{2}, \delta_{1}>0$. The tangent to the hypersurface $S_{2}$ at a point $\boldsymbol{x} \in S_{2}$ vector $\boldsymbol{\tau}(\boldsymbol{x})=\left(1, \tau_{1}(\boldsymbol{x}), \ldots, \tau_{n}(\boldsymbol{x})\right)$ satisfies the conditions

$$
\left|\tau_{i}\right| \leqslant \delta_{2}, \quad\left|\frac{\partial \tau_{i}}{\partial x_{j}}\right| \leqslant \delta_{2}, \quad i=1, \ldots, n, j=0, \ldots, n,
$$

where $\delta_{2}$ is a sufficiently small positive number. For equation (2.1) we formulate the following boundary conditions:

$$
\left.u\right|_{S_{1}}=\left.\frac{\partial u}{\partial \boldsymbol{p}_{1}}\right|_{S_{1}}=\left.\frac{\partial^{2} u}{\partial \boldsymbol{p}_{1}^{2}}\right|_{S_{1}}=0
$$




$$
\begin{gathered}
\left.u\right|_{S_{2}}=\left.\frac{\partial u}{\partial \boldsymbol{p}_{2}}\right|_{S_{2}}=0, \\
\left.u\right|_{S_{3}}=0,
\end{gathered}
$$

where $\frac{\partial}{\partial \boldsymbol{p}_{i}}$ is derivative in the direction of $\boldsymbol{p}_{i}$ from vector field $\mathcal{P}_{i}$, which is not tangent to $S_{i}, i=1,2$. If boundary conditions (2.3)-(2.5) are nonhomogeneous, then they are reduced to the homogeneous ones by extending their right parts to the domain $Q$ by functions from suitable spaces and replacing the desired function [23].

\section{Definition of Weak Solution}

We consider problem (2.1)-(2.5) and define some functional spaces in which a weak solution is defined. Weak solution is defined by some equality of the corresponding functionals. To achieve this, along with problem (2.1)-(2.5) we consider an adjoint boundary value problem:

$$
\begin{aligned}
\mathcal{L}^{\prime} v & \equiv \frac{\partial^{4} v}{\partial x_{0}^{4}}+\left(b^{2}-a^{2}\right) \frac{\partial^{2}}{\partial x_{0}^{2}} \Delta v-a^{2} b^{2} \Delta^{2} v+A^{(2) \prime} v=g(\boldsymbol{x}), \\
\left.v\right|_{S_{1}} & =0 \\
\left.v\right|_{S_{2}} & =\left.\frac{\partial v}{\partial \boldsymbol{p}_{2}}\right|_{S_{2}}=0 \\
\left.v\right|_{S_{3}} & =\left.\frac{\partial v}{\partial \boldsymbol{p}_{3}}\right|_{S_{3}}=\left.\frac{\partial^{2} v}{\partial \boldsymbol{p}_{3}^{2}}\right|_{S_{3}}=0,
\end{aligned}
$$

where $\frac{\partial}{\partial \boldsymbol{p}_{3}}$ is derivative in the direction of $\boldsymbol{p}_{3}$ from vector field $\mathcal{P}_{3}$, which is not tangent to $S_{3}, A^{(2) \prime} v=\sum_{|\boldsymbol{\alpha}| \leqslant 2}(-1)^{|\boldsymbol{\alpha}|} \mathbf{D}^{\boldsymbol{\alpha}}\left(a^{(\boldsymbol{\alpha})}(\boldsymbol{x}) v\right)$.

We define domains of definitions for the operators $\mathcal{L}$ and $\mathcal{L}^{\prime}$ as follows:

$$
\begin{aligned}
\mathcal{D}(\mathcal{L}) & =\left\{u \in C^{4}(\bar{Q})|u|_{S_{1}}=\left.\frac{\partial u}{\partial \boldsymbol{p}_{1}}\right|_{S_{1}}=\left.\frac{\partial^{2} u}{\partial \boldsymbol{p}_{1}^{2}}\right|_{S_{1}}=\left.u\right|_{S_{2}}=\left.\frac{\partial u}{\partial \boldsymbol{p}_{2}}\right|_{S_{2}}=\left.u\right|_{S_{3}}=0\right\}, \\
\mathcal{D}\left(\mathcal{L}^{\prime}\right) & =\left\{v \in C^{4}(\bar{Q})|v|_{S_{1}}=\left.v\right|_{S_{2}}=\left.\frac{\partial v}{\partial \boldsymbol{p}_{2}}\right|_{S_{2}}=\left.v\right|_{S_{3}}=\left.\frac{\partial v}{\partial \boldsymbol{p}_{3}}\right|_{S_{3}}=\left.\frac{\partial^{2} v}{\partial \boldsymbol{p}_{3}^{2}}\right|_{S_{3}}=0\right\} .
\end{aligned}
$$

It is easy to check that

$$
(\mathcal{L} u, v)_{L_{2}(Q)}=\left(u, \mathcal{L}^{\prime} v\right)_{L_{2}(Q)}
$$

for any functions $u \in \mathcal{D}(\mathcal{L})$ and $v \in \mathcal{D}\left(\mathcal{L}^{\prime}\right)$, where $(\cdot, \cdot)_{L_{2}(Q)}$ is value of scalar product in the space $L_{2}(Q)$ of square integrable in $Q$ functions.

We denote by $H^{l}(Q)$ Hilbert space elements of which $u \in L_{2}(Q)$ and their weak derivatives $\mathbf{D}^{\boldsymbol{\alpha}} u,|\boldsymbol{\alpha}| \leqslant l$, belong to $L_{2}(Q)$. A scalar product in $H^{l}(Q)$ is defined by expression $(u, v)_{H^{l}(Q)}=\sum_{|\boldsymbol{\alpha}| \leqslant l}\left(\mathbf{D}^{\boldsymbol{\alpha}} u, \mathbf{D}^{\boldsymbol{\alpha}} v\right)_{L_{2}(Q)}$. Let $H_{0}^{l}(Q)(l=$ $1,2,3)$ be a subspace of the space $H^{l}(Q)$ elements of which satisfy boundary conditions (2.3)-(2.5) according to the S. L. Sobolev embedding theorems. In 
a similar way, subspaces $H^{l}(Q)$ of the space $H^{l}(Q)$ are defined, where their elements satisfy conditions (3.1)-(3.3).

Condition 1. The boundary $\partial Q$ of the domain $Q$ is such that the closure of the set $\mathcal{D}(\mathcal{L})$ in the norm of the space $H^{l}(Q)$ is the same as the space $H_{0}^{l}(Q)$ and the closure of the set $\mathcal{D}\left(\mathcal{L}^{\prime}\right)$ is the same as the space $\stackrel{0}{H}^{l}(Q), l=1,2,3$.

Domains with sufficiently smooth boundaries satisfy Condition 1.

We remark that the elements of the space $H_{0}^{2}(Q)$ have meaning not for all boundary conditions (2.3)-(2.5). If $u \in H_{0}^{2}(Q)$, then $u$ actually satisfies conditions (2.4), (2.5) and first two conditions from (2.3). If $u \in H_{0}^{1}(Q)$, then $\left.u\right|_{\partial Q}=0$. A similar remark takes place for the elements $v \in \stackrel{0}{H}^{l}(Q)(l=1,2)$ with respect to conditions (3.1)-(3.3). Here according to introduced notation and definitions of the spaces $H_{0}^{1}(Q)=\stackrel{0}{H}^{1}(Q)$. We remark that the subspaces $H_{0}^{l}(Q)$ and $\stackrel{0}{H}^{l}(Q), l=1,2,3$, are Hilbert spaces, too.

We denote by $H_{0}^{-1}$ and $\stackrel{0}{H}^{-1}$ spaces with negative norm [2] with respect to the spaces $H_{0}^{1}(Q)$ and $\stackrel{0}{H}^{1}(Q)$, i.e. spaces of all continuous linear functionals over the spaces $H_{0}^{1}(Q)$ and $\stackrel{0}{H}^{1}(Q)$. As far as $H_{0}^{1}(Q)=\stackrel{0}{H}^{1}(Q)$, then $H_{0}^{-1}=\stackrel{0}{H^{-1}}$.

To define weak solution of problem (2.1)-(2.5) we consider bilinear form

$$
\Phi(u, v)=\left(u, \mathcal{L}^{\prime} v\right)_{L_{2}(Q)}, \quad u \in \mathcal{D}(\mathcal{L}), v \in \mathcal{D}\left(\mathcal{L}^{\prime}\right)
$$

If $u \in H_{0}^{2}(Q)$ and $v \in \stackrel{0}{H}^{3}(Q)$, then the form $\Phi(u, v)$ can be written in the form

$$
\begin{aligned}
\Phi(u, v)= & \left(\frac{\partial^{2} u}{\partial x_{0}^{2}}, \frac{\partial^{2} v}{\partial x_{0}^{2}}\right)_{L_{2}(Q)}+\left(b^{2}-a^{2}\right) \sum_{i=1}^{n}\left(\frac{\partial^{2} u}{\partial x_{0} \partial x_{i}}, \frac{\partial^{2} v}{\partial x_{0} \partial x_{i}}\right)_{L_{2}(Q)} \\
& -a^{2} b^{2} \sum_{i, j=1}^{n}\left(\frac{\partial^{2} u}{\partial x_{i} \partial x_{j}}, \frac{\partial^{2} v}{\partial x_{i} \partial x_{j}}\right)_{L_{2}(Q)}+\left(A^{(2)} u, v\right)_{L_{2}(Q)} .
\end{aligned}
$$

For every $u \in H_{0}^{2}(Q)$ we consider $\Phi(u, v)$ as a linear functional $v \rightarrow \Phi(u, v)$.

We define the extension $\mathcal{L}$ of the operator $\mathcal{L}$ in the following way by taking into account (3.4). A function $u$ belongs to the domain of definition $\mathcal{D}(\mathcal{L})$ of the operator $\mathcal{L}$, if $u \in H_{0}^{2}(Q)$ and the map $v \rightarrow \Phi(u, v)$ is linear continuous functional in the dense set $H^{3}(Q)$ of the space $H_{0}^{1}(Q)$ in topology induced from the space $H_{0}^{1}(Q)$. Then this functional allows a continuous extension to all space $H_{0}^{1}(Q)$. Consequently, a unique element $\mathcal{L} u \in H_{0}^{-1}$ exists such that

$$
\Phi(u, v)=\langle\mathcal{L} u, v\rangle
$$

for $u \in \mathcal{D}(\mathcal{L})$ and any $v \in H_{0}^{1}(Q)$ exists, where $\langle w, v\rangle$ is canonical bilinear form for $w \in H_{0}^{-1}$ and $v \in H_{0}^{1}(Q)$, which is extension by continuity of the bilinear form $(w, v)_{L_{2}(Q)}$, where $w \in L_{2}(Q), v \in H_{0}^{1}(Q)$. 
As far as the set $\stackrel{0}{H}^{3}(Q)$ is dense in the space $H_{0}^{1}(Q)$, then

$$
\|\mathcal{L} u\|_{H_{0}^{-1}}=\sup _{\substack{0 \\ v \in H^{3}(Q)}} \frac{|\Phi(u, v)|}{\|v\|_{H_{0}^{1}(Q)}}, \quad u \in \mathcal{D}(\mathcal{L}) .
$$

It follows from (3.4) and (3.5) that if $u \in \mathcal{D}(\mathcal{L})$, then $u \in \mathcal{D}(\mathcal{L})$. Really, $\Phi(u, v)=(\mathcal{L} u, v)_{L_{2}(Q)}$ and is linear continuous functional with respect to $v$ for $u \in \mathcal{D}(\mathcal{L}), v \in H_{0}^{1}(Q)$, i.e. $\mathcal{L} u=\mathcal{L} u$ for $u \in \mathcal{D}(\mathcal{L})$. Consequently, $\mathcal{L}$ is extension of the operator $\mathcal{L}$.

Definition 1. The solution of operator equation $\mathcal{L} u=f, f \in H_{0}^{-1}$, is a weak solution of problem (2.1)-(2.5).

In a similar way, starting from (3.4), we build the extension $\mathcal{L}^{\prime}$ of the operator $\mathcal{L}^{\prime}$. A function $v$ belongs to the domain of definition $\mathcal{D}\left(\mathcal{L}^{\prime}\right)$ of the operator $\mathcal{L}^{\prime}$, if $v \in \stackrel{0}{H}^{2}(Q)$ and a map $u \rightarrow \Psi(u, v)$, where

$$
\begin{aligned}
\Psi(u, v)= & \left(\frac{\partial^{2} u}{\partial x_{0}^{2}}, \frac{\partial^{2} v}{\partial x_{0}^{2}}\right)_{L_{2}(Q)}+\left(b^{2}-a^{2}\right) \sum_{i=1}^{n}\left(\frac{\partial^{2} u}{\partial x_{0} \partial x_{i}}, \frac{\partial^{2} v}{\partial x_{0} \partial x_{i}}\right)_{L_{2}(Q)} \\
& -a^{2} b^{2} \sum_{i, j=1}^{n}\left(\frac{\partial^{2} u}{\partial x_{i} \partial x_{j}}, \frac{\partial^{2} v}{\partial x_{i} \partial x_{j}}\right)_{L_{2}(Q)}+\left(A^{(2)} u, v\right)_{L_{2}(Q)},
\end{aligned}
$$

$u \in H_{0}^{3}(Q)$, is linear continuous functional in the dense set $H_{0}^{3}(Q)$ of the space $H_{0}^{1}(Q)$ in topology induced from the space $H_{0}^{1}(Q)$. Then this functional allows continuous extension to space $H_{0}^{1}(Q)$. Consequently, a unique element $\mathcal{L}^{\prime} v \in$ $H_{0}^{-1}$ exists, such that $\Psi(u, v)=\left\langle u, \mathcal{L}^{\prime} v\right\rangle$ for $v \in \mathcal{D}\left(\mathcal{L}^{\prime}\right)$ and any $u \in H_{0}^{1}(Q)$, where $\langle u, w\rangle$ is canonical bilinear form for $u \in H_{0}^{1}(Q)$ and $w \in H_{0}^{-1}$, which is extension by continuity of the bilinear form $(u, w)_{L_{2}(Q)}$, where $u \in H_{0}^{1}(Q)$ and $w \in L_{2}(Q)$. As far as the set $H_{0}^{3}(Q)$ is dense in the space $H_{0}^{1}(Q)$, then

$$
\left\|\mathcal{L}^{\prime} v\right\|_{H_{0}^{-1}}=\sup _{u \in H_{0}^{3}(Q)} \frac{|\Psi(u, v)|}{\|u\|_{H_{0}^{1}(Q)}}, \quad v \in \mathcal{D}\left(\mathcal{L}^{\prime}\right) .
$$

It follows from (3.4) and (3.7) that if $v \in \mathcal{D}\left(\mathcal{L}^{\prime}\right)$, then $v \in \mathcal{D}\left(\mathcal{L}^{\prime}\right)$. Comparing (3.5) and (3.7), we conclude that the following equality holds:

$$
\langle\mathcal{L} u, v\rangle=\left\langle u, \mathcal{L}^{\prime} v\right\rangle, \quad u \in H_{0}^{3}(Q), v \in \stackrel{0}{H}^{3}(Q) .
$$

\section{Existence and Uniqueness of the Weak Solution}

Existence of the weak solution is proved on the basis of (3.8), if corresponding a priori estimations for the operators $\mathcal{L}$ and $\mathcal{L}^{\prime}$ are obtained. Here we take into account that the spaces $H_{0}^{2}(Q)$ and $\stackrel{0}{H}^{2}(Q)$ are reflexive. 
Theorem 1. The inequalities

$$
\begin{aligned}
\|u\|_{H_{0}^{2}(Q)} & \leqslant c_{1}\|\mathcal{L} u\|_{H_{0}^{-1}}, \quad u \in \mathcal{D}(\mathcal{L}), \\
\|v\|_{H^{2}(Q)} \leqslant c_{2}\left\|\mathcal{L}^{\prime} v\right\|_{H_{0}^{-1}}, & v \in \mathcal{D}\left(\mathcal{L}^{\prime}\right)
\end{aligned}
$$

are valid, where $c_{i}$ are some positive constants independent of $u$ and $v$.

Proof. We prove that inequality (4.1) is correct. We suppose at first $u \in$ $H_{0}^{2}(Q) \cap H^{3}(Q)$. It follows from (3.5) that in order that the functional $v \rightarrow$ $\Phi(u, v)$ is continuous over the space $H_{0}^{1}(Q)$, the equality $\left.\frac{\partial^{2} u}{\partial \boldsymbol{p}_{1}^{2}}\right|_{S_{1}}=0$ must be satisfied, i.e. actually $u \in H_{0}^{3}(Q)$, if $u \in \mathcal{D}(\mathcal{L})$. In this case the value of the functional $\Phi(u, v)$ can be represented in the form

$$
\begin{aligned}
\Phi(u, v)= & -\left(\frac{\partial^{3} u}{\partial x_{0}^{3}}, \frac{\partial v}{\partial x_{0}}\right)_{L_{2}(Q)}-\left(b^{2}-a^{2}\right) \sum_{i=1}^{n}\left(\frac{\partial^{3} u}{\partial x_{0}^{2} \partial x_{i}}, \frac{\partial v}{\partial x_{i}}\right)_{L_{2}(Q)} \\
& +a^{2} b^{2} \sum_{i, j=1}^{n}\left(\frac{\partial^{3} u}{\partial x_{i}^{2} \partial x_{j}}, \frac{\partial v}{\partial x_{j}}\right)_{L_{2}(Q)}+\left(A^{(2)} u, v\right)_{L_{2}(Q)}, \quad v \in H^{3}(Q) .
\end{aligned}
$$

The functional $\Phi(u, v)$ written in form (4.3) is extended by continuity to all space $H_{0}^{1}(Q)$. We suppose in (4.3) $v=\mathcal{M} u$, where $\mathcal{M} u=\left(x_{0}-T\right) \frac{\partial u}{\partial \boldsymbol{\tau}}-u$. Such $v \in H_{0}^{1}(Q)$ by virtue of $(2.3)-(2.5)$. In this case

$$
\begin{aligned}
\Phi(u, \mathcal{M} u) & =\int_{Q}\left(-\frac{\partial^{3} u}{\partial x_{0}^{3}} \frac{\partial u}{\partial \boldsymbol{\tau}}-\frac{\partial^{3} u}{\partial x_{0}^{3}}\left(x_{0}-T\right) \frac{\partial^{2} u}{\partial \boldsymbol{\tau} \partial x_{0}}+\frac{\partial^{3} u}{\partial x_{0}^{3}} \frac{\partial u}{\partial x_{0}}+A^{(2)} u \mathcal{M} u\right. \\
& -\left(b^{2}-a^{2}\right) \sum_{i=1}^{n} \frac{\partial^{3} u}{\partial x_{0}^{2} \partial x_{i}}\left(x_{0}-T\right) \frac{\partial^{2} u}{\partial \boldsymbol{\tau} \partial x_{i}}+\left(b^{2}-a^{2}\right) \sum_{i=1}^{n} \frac{\partial^{3} u}{\partial x_{0}^{2} \partial x_{i}} \frac{\partial u}{\partial x_{i}} \\
& \left.+a^{2} b^{2} \sum_{i, j=1}^{n} \frac{\partial^{3} u}{\partial x_{i}^{2} \partial x_{j}}\left(x_{0}-T\right) \frac{\partial^{2} u}{\partial \boldsymbol{\tau} \partial x_{j}}-a^{2} b^{2} \sum_{i, j=1}^{n} \frac{\partial^{3} u}{\partial x_{i}^{2} \partial x_{j}} \frac{\partial u}{\partial x_{j}}\right) \mathrm{d} \boldsymbol{x} .
\end{aligned}
$$

We represent the main part of the expression under the integral sign in the last relation in the divergent form:

$$
\Phi(u, \mathcal{M} u)=I_{1}+I_{2}+I_{3}+I_{4},
$$

where

$$
\begin{aligned}
I_{1} & =\int_{Q}\left(-\sum_{i=1}^{n} \frac{\partial}{\partial x_{0}}\left(\frac{\partial^{2} u}{\partial x_{0}^{2}} \tau_{i} \frac{\partial u}{\partial x_{i}}\right)-\frac{\partial}{\partial x_{0}}\left(\frac{\partial^{2} u}{\partial x_{0}^{2}}\left(x_{0}-T\right) \frac{\partial^{2} u}{\partial \tau \partial x_{0}}\right)\right. \\
& -\left(b^{2}-a^{2}\right) \sum_{i=1}^{n}\left[\frac{\partial}{\partial x_{0}}\left(\frac{\partial^{2} u}{\partial x_{0} \partial x_{i}}\left(x_{0}-T\right) \frac{\partial^{2} u}{\partial \tau \partial x_{i}}\right)-\frac{\partial}{\partial x_{0}}\left(\frac{\partial^{2} u}{\partial x_{0} \partial x_{i}} \frac{\partial u}{\partial x_{i}}\right)\right] \\
& +a^{2} b^{2} \sum_{i, j=1}^{n} \frac{\partial}{\partial x_{i}}\left(\frac{\partial^{2} u}{\partial x_{i} \partial x_{j}}\left(x_{0}-T\right) \frac{\partial^{2} u}{\partial \tau \partial x_{j}}\right)-a^{2} b^{2} \sum_{i, j=1}^{n} \frac{\partial}{\partial x_{i}}\left(\frac{\partial^{2} u}{\partial x_{i} \partial x_{j}} \frac{\partial u}{\partial x_{j}}\right)
\end{aligned}
$$




$$
\begin{aligned}
& +\frac{1}{2} \frac{\partial}{\partial \boldsymbol{\tau}}\left(\left(x_{0}-T\right)\left(\frac{\partial^{2} u}{\partial x_{0}^{2}}\right)^{2}\right)+\frac{b^{2}-a^{2}}{2} \sum_{i=1}^{n} \frac{\partial}{\partial \boldsymbol{\tau}}\left(\left(x_{0}-T\right)\left(\frac{\partial^{2} u}{\partial x_{0} \partial x_{i}}\right)^{2}\right) \\
& \left.-\frac{a^{2} b^{2}}{2} \sum_{i, j=1}^{n} \frac{\partial}{\partial \boldsymbol{\tau}}\left(\left(x_{0}-T\right)\left(\frac{\partial^{2} u}{\partial x_{i} \partial x_{j}}\right)^{2}\right)\right) \mathrm{d} \boldsymbol{x} \\
I_{2}= & \int_{Q}\left(\frac{1}{2}\left(\frac{\partial^{2} u}{\partial x_{0}^{2}}\right)^{2}-\frac{b^{2}-a^{2}}{2} \sum_{i=1}^{n}\left(\frac{\partial^{2} u}{\partial x_{0} \partial x_{i}}\right)^{2}+\frac{3 a^{2} b^{2}}{2} \sum_{i, j=1}^{n}\left(\frac{\partial^{2} u}{\partial x_{i} \partial x_{j}}\right)^{2}\right) \mathrm{d} \boldsymbol{x}, \\
I_{3}= & \int_{Q}\left(2 \sum_{i=1}^{n} \frac{\partial^{2} u}{\partial x_{0}^{2}} \tau_{i} \frac{\partial^{2} u}{\partial x_{0} \partial x_{i}}+2 \sum_{i=1}^{n} \frac{\partial^{2} u}{\partial x_{0}^{2}}\left(x_{0}-T\right) \frac{\partial \tau_{i}}{\partial x_{0}} \frac{\partial^{2} u}{\partial x_{0} \partial x_{i}}\right. \\
& +\left(b^{2}-a^{2}\right) \sum_{i, j=1}^{n} \frac{\partial^{2} u}{\partial x_{0} \partial x_{i}}\left[\tau_{j} \frac{\partial^{2} u}{\partial x_{i} \partial x_{j}}+\left(x_{0}-T\right) \frac{\partial \tau_{j}}{\partial x_{0}} \frac{\partial^{2} u}{\partial x_{i} \partial x_{j}}\right] \\
& +\left(b^{2}-a^{2}\right) \sum_{i, j=1}^{n} \frac{\partial^{2} u}{\partial x_{0} \partial x_{i}}\left(x_{0}-T\right) \frac{\partial \tau_{j}}{\partial x_{i}} \frac{\partial^{2} u}{\partial x_{0} \partial x_{j}} \\
& \left.-a^{2} b^{2} \sum_{i, j, k=1}^{n} \frac{\partial^{2} u}{\partial x_{i} \partial x_{j}}\left(x_{0}-T\right) \frac{\partial^{2} \tau_{k}}{\partial x_{i} \partial x_{j}} \frac{\partial u}{\partial x_{k}}\right) \mathrm{d} \boldsymbol{x} . \\
& \left.-2 a^{2} b^{2} \sum_{i, j, k=1}^{n} \frac{\partial^{2} u}{\partial x_{i} \partial x_{j}}\left(x_{0}-T\right) \frac{\partial \tau_{k}}{\partial x_{i}} \frac{\partial^{2} u}{\partial x_{j} \partial x_{k}}\right) \mathrm{d} \boldsymbol{x}, \\
I_{4}= & \int_{Q}\left(A^{(2)} u \mathcal{M} u+2 \sum_{i=1}^{n} \frac{\partial^{2} u}{\partial x_{0}^{2}} \frac{\partial \tau_{i}}{\partial x_{0}} \frac{\partial u}{\partial x_{i}}+\sum_{i=1}^{n} \frac{\partial^{2} u}{\partial x_{0}^{2}}\left(x_{0}-T\right) \frac{\partial^{2} \tau_{i}}{\partial x_{0}^{2}} \frac{\partial u}{\partial x_{i}}\right. \\
& \left.+\left(b^{2}-a^{2}\right) \sum_{j}^{n} \frac{\partial \tau_{j}}{\partial x_{i}} \frac{\partial u}{\partial x_{j}}+\left(x_{0}-T\right) \frac{\partial u}{\partial x_{0} \partial x_{i}}\right] \\
& \left(x_{j}\right] \\
& \\
&
\end{aligned}
$$

We apply the Ostrogradskii formula to $I_{1}$ and get $I_{1}=0$ by virtue of $(2.3)$ (2.5). With the help of the inequality

$$
2|a b| \leqslant \varepsilon a^{2}+\frac{1}{\varepsilon} b^{2}, \quad \forall \varepsilon>0,
$$

and conditions (2.2) we get the upper bound for $I_{3}$. For $I_{4}$ the upper bound is obtained with the help of the Cauchy-Bunyakovsky inequality and conditions (2.2):

$$
\left|I_{4}\right| \leqslant c_{3}\|u\|_{H_{0}^{2}(Q)}\|u\|_{H_{0}^{1}(Q)} .
$$

In this way we have the lower bound for the functional $\Phi(u, \mathcal{M} u)$ :

$$
\Phi(u, \mathcal{M} u) \leqslant c_{4}\|u\|_{H_{0}^{2}(Q)}^{2}-c_{5}\|u\|_{H_{0}^{2}(Q)}\|u\|_{H_{0}^{1}(Q)}
$$

As far as $\|\mathcal{M} u\|_{H_{0}^{1}(Q)} \leqslant c_{6}\|u\|_{H_{0}^{2}(Q)}$, taking into account (3.6) and (4.5) we get

$$
\|\mathcal{L} u\|_{H_{0}^{-1}} \leqslant c_{7}\|u\|_{H_{0}^{2}(Q)}-c_{8}\|u\|_{H_{0}^{1}(Q)} .
$$


We integrate over domain $Q^{(\tau)}=\left\{\boldsymbol{x} \in Q \mid 0<x_{0}<\tau<T\right\}, T=\sup _{\boldsymbol{x} \in Q} x_{0}$, the identity

$$
c_{9} \sum_{|\boldsymbol{\alpha}| \leqslant 1} \frac{\partial}{\partial x_{0}}\left(\mathbf{D}^{\boldsymbol{\alpha}} u\right)^{2}=2 c_{9} \sum_{|\boldsymbol{\alpha}| \leqslant 1} \mathbf{D}^{\boldsymbol{\alpha}} u \frac{\partial}{\partial x_{0}} \mathbf{D}^{\boldsymbol{\alpha}} u,
$$

where $c_{9}$ is sufficiently large positive constant:

$$
\begin{aligned}
& c_{9} \int_{S_{3}^{(\tau)}} \sum_{|\boldsymbol{\alpha}| \leqslant 1}\left(\mathbf{D}^{\boldsymbol{\alpha}} u\right)^{2}\left(\tau, \boldsymbol{x}^{\prime}\right) \mathrm{d} \boldsymbol{x}^{\prime}=2 c_{9} \int_{Q^{(\tau)}} \sum_{|\boldsymbol{\alpha}| \leqslant 1} \mathbf{D}^{\boldsymbol{\alpha}} u \frac{\partial}{\partial x_{0}} \mathbf{D}^{\boldsymbol{\alpha}} u \mathrm{~d} \boldsymbol{x} \\
& \quad \leqslant \frac{c_{9}}{\varepsilon} \int_{Q^{(\tau)}} \sum_{|\boldsymbol{\alpha}| \leqslant 1}\left(\mathbf{D}^{\boldsymbol{\alpha}} u\right)^{2} \mathrm{~d} \boldsymbol{x}+\varepsilon c_{9} \int_{Q^{(\tau)}} \sum_{|\boldsymbol{\alpha}| \leqslant 1}\left(\frac{\partial}{\partial x_{0}} \mathbf{D}^{\boldsymbol{\alpha}} u\right)^{2} \mathrm{~d} \boldsymbol{x} \\
& \quad \leqslant \frac{c_{9}}{\varepsilon} \int_{Q^{(\tau)}} \sum_{|\boldsymbol{\alpha}| \leqslant 1}\left(\mathbf{D}^{\boldsymbol{\alpha}} u\right)^{2} \mathrm{~d} \boldsymbol{x}+\varepsilon c_{9}\|u\|_{H_{0}^{2}(Q)}^{2},
\end{aligned}
$$

$\partial Q^{(\tau)}=S_{1} \cup S_{2}^{(\tau)} \cup S_{3}^{(\tau)}, S_{2}^{(\tau)}=\left\{\boldsymbol{x} \in S_{2} \mid 0<x_{0}<\tau\right\}, S_{3}^{(\tau)}=\{\boldsymbol{x} \in$ $\left.Q \mid x_{0}=\tau\right\}$. We have used inequality (4.4) in (4.7). Let denote by $v(\tau)=$ $c_{9} \int_{S_{3}^{(\tau)}} \sum_{|\boldsymbol{\alpha}| \leqslant 1}\left(\mathbf{D}^{\boldsymbol{\alpha}} u\right)^{2}\left(\tau, \boldsymbol{x}^{\prime}\right) \mathrm{d} \boldsymbol{x}^{\prime}$. Then

$$
c_{9} \int_{Q^{(\tau)}} \sum_{|\boldsymbol{\alpha}| \leqslant 1}\left(\mathbf{D}^{\boldsymbol{\alpha}} u\right)^{2} \mathrm{~d} \boldsymbol{x}=\int_{0}^{\tau} v(t) \mathrm{d} t
$$

and inequality (4.7) takes the form $v(\tau) \leqslant \frac{1}{\varepsilon} \int_{0}^{\tau} v(t) \mathrm{d} t+\varepsilon c_{9}\|u\|_{H_{0}^{2}(Q)}$. We add it to inequality (4.6), choose $\varepsilon$ such that the inequality $c_{7}-\varepsilon c_{9}>0$ is satisfied and apply the Gronwall inequality:

$$
\begin{aligned}
c_{10}\|u\|_{H_{0}^{2}(Q)}+v(\tau) & \leqslant \mathrm{e}^{\tau / \varepsilon}\left(\|\mathcal{L} u\|_{H_{0}^{-1}}+c_{8}\|u\|_{H_{0}^{1}(Q)}\right) \\
& \leqslant \mathrm{e}^{T / \varepsilon}\|\mathcal{L} u\|_{H_{0}^{-1}}+\mathrm{e}^{\frac{T}{\varepsilon}} c_{8}\|u\|_{H_{0}^{1}(Q)}
\end{aligned}
$$

The right part of inequality (4.8) is independent of $\tau$, therefore in its left part one can pass to the least upper bound with respect to $\tau$. We get the following inequality:

$$
c_{10}\|u\|_{H_{0}^{2}(Q)}+c_{9} \sup _{0<\tau<T}\|u\|_{H_{0}^{1}\left(S_{3}^{(\tau)}\right)}^{2}(\tau) \leqslant e^{\frac{T}{\varepsilon}}\|\mathcal{L} u\|_{H_{0}^{-1}}+e^{\frac{T}{\varepsilon}} c_{8}\|u\|_{H_{0}^{1}(Q)} .
$$

Taking into account estimate $\sup _{0<\tau<T}\|u\|_{H_{0}^{1}\left(S_{3}^{(\tau)}\right)}^{2}(\tau) \leqslant \frac{1}{T}\|u\|_{H_{0}^{1}(Q)}^{2}$ the inequality $\|u\|_{H_{0}^{2}(Q)} \leqslant c_{11}\|\mathcal{L} u\|_{H_{0}^{-1}}$ is proved in case $u \in H_{0}^{2}(Q) \cap H^{3}(Q)$.

Now let $u \in \mathcal{D}(\mathcal{L})$. Then $J_{k} u \in H_{0}^{2}(Q) \cap H^{3}(Q)$, where $J_{k}$ is mollifier with variable step $[3,16,17]$. It is proved above that

$$
\left\|J_{k} u\right\|_{H_{0}^{2}(Q)} \leqslant \sup _{\substack{0 \\ v \in H^{3}(Q)}} \frac{\left|\Phi\left(J_{k} u, v\right)\right|}{\|v\|_{H_{0}^{1}(Q)}} .
$$

We represent the functional $\Phi\left(J_{k} u, v\right)$ in the following form:

$$
\Phi\left(J_{k} u, v\right)=\Phi\left(u, J_{k}^{*} v\right)+K_{1}(u, v ; k)+K_{2}(u, v ; k),
$$


where

$$
\begin{aligned}
K_{1}(u, v ; k)= & \left(\frac{\partial^{3}}{\partial x_{0}^{3}} J_{k} u-\frac{\partial}{\partial x_{0}} J_{k} \frac{\partial^{2} u}{\partial x_{0}^{2}}, \frac{\partial v}{\partial x_{0}}\right)_{L_{2}(Q)} \\
& +\left(b^{2}-a^{2}\right) \sum_{i=1}^{n}\left(\frac{\partial^{3}}{\partial x_{0}^{2} \partial x_{i}} J_{k} u-\frac{\partial}{\partial x_{0}} J_{k} \frac{\partial^{2} u}{\partial x_{0} \partial x_{i}}, \frac{\partial v}{\partial x_{i}}\right)_{L_{2}(Q)} \\
& -a^{2} b^{2} \sum_{i, j=1}^{n}\left(\frac{\partial^{3}}{\partial x_{i}^{2} \partial x_{j}} J_{k} u-\frac{\partial}{\partial x_{i}} J_{k} \frac{\partial^{2} u}{\partial x_{i} \partial x_{j}}, \frac{\partial v}{\partial x_{j}}\right)_{L_{2}(Q)} \\
& +\left(A^{(2)} J_{k} u-J_{k} A^{(2)} u, v\right)_{L_{2}(Q)}, \\
K_{2}(u, v ; k)= & \left(\frac{\partial^{2} u}{\partial x_{0}^{2}}, J_{k}^{*} \frac{\partial^{2} v}{\partial x_{0}^{2}}-\frac{\partial^{2}}{\partial x_{0}^{2}} J_{k}^{*} v\right)_{L_{2}(Q)} \\
& +\left(b^{2}-a^{2}\right) \sum_{i=1}^{n}\left(\frac{\partial^{2} u}{\partial x_{0} \partial x_{i}}, J_{k}^{*} \frac{\partial^{2} v}{\partial x_{0} \partial x_{i}}-\frac{\partial^{2}}{\partial x_{0} \partial x_{i}} J_{k}^{*} v\right)_{L_{2}(Q)} \\
& -a^{2} b^{2} \sum_{i, j=1}^{n}\left(\frac{\partial^{2} u}{\partial x_{i} \partial x_{j}}, J_{k}^{*} \frac{\partial^{2} v}{\partial x_{i} \partial x_{j}}-\frac{\partial^{2}}{\partial x_{i} \partial x_{j}} J_{k}^{*} v\right)_{L_{2}(Q)} .
\end{aligned}
$$

We have used here the following assumptions: in order that functional $v \rightarrow$ $\Phi(u, v)$ be continuous over the space $H_{0}^{1}(Q)$, the equality $\left.J_{k} \frac{\partial^{2} u}{\partial \boldsymbol{p}_{1}^{2}}\right|_{\Omega^{(0)}}=0$ must be satisfied, and $\left.\frac{\partial^{2}}{\partial p_{1}^{2}} J_{k} u\right|_{\Omega^{(0)}}=0$ was proved above. By virtue of the properties of the mollifiers $J_{k}$ and $J_{k}^{*}$ we get

$$
\begin{aligned}
& K_{1}(u, v ; k), K_{2}(u, v ; k) \leqslant \frac{1}{k}\|u\|_{H_{0}^{2}(Q)}\|v\|_{H_{0}^{1}(Q)} \\
& \left\|J_{k}^{*} v\right\|_{H_{0}^{1}(Q)} \leqslant c_{12}\|v\|_{H_{0}^{1}(Q)} .
\end{aligned}
$$

By virtue of estimates (4.10), (4.11) inequality (4.9) can be continued in the following way:

$$
\begin{aligned}
& \left\|J_{k} u\right\|_{H_{0}^{2}(Q)} \leqslant \sup _{\substack{0 \in H^{3}(Q) \\
v \in H^{0}}} \frac{\left|\Phi\left(J_{k} u, v\right)\right|}{\|v\|_{H_{0}^{1}(Q)}} \leqslant \sup _{\substack{0 \in H^{3}(Q) \\
v \in H^{0}}} \frac{\left|\Phi\left(u, J_{k}^{*} v\right)\right|}{\|v\|_{H_{0}^{1}(Q)}}+\frac{2}{k}\|u\|_{H_{0}^{2}(Q)} \\
& \leqslant c_{12} \sup _{\substack{v \in H^{3}(Q) \\
\text { on }}} \frac{\left|\Phi\left(u, J_{k}^{*} v\right)\right|}{\left\|J_{k}^{*} v\right\|_{H_{0}^{1}(Q)}}+\frac{2}{k}\|u\|_{H_{0}^{2}(Q)} \\
& \leqslant c_{12} \sup _{\substack{0 \\
v H^{3}(Q)}} \frac{|\Phi(u, v)|}{\|v\|_{H_{0}^{1}(Q)}}+\frac{2}{k}\|u\|_{H_{0}^{2}(Q)} .
\end{aligned}
$$

Passing in it to the limit as $k \rightarrow \infty$, we get desired inequality (4.1). Inequality (4.2) is proved in the same way.

Theorem 2. If Condition 1 is satisfied and $f \in H_{0}^{-1}$, then a unique weak solution of problem (2.1)-(2.5) exists. 
Proof. Uniqueness of weak solution of problem (2.1)-(2.5) follows from Theorem 1. The operator $\mathcal{L}$ is closed, therefore for proving existence of weak solution of problem (2.1)-(2.5) and, thus, for finishing the proof of the theorem it remains to prove that $\mathcal{R}(\mathcal{L})=H_{0}^{-1}$. It is enough to show the density of the elements $\mathcal{L} u$, where $u \in H_{0}^{3}(Q)$, is in the space $H_{0}^{-1}$. Let $v \in \stackrel{0}{H}^{3}(Q)$ be such that for any function $u$ from the indicated class $\langle\mathcal{L} u, v\rangle=0$. As $u \in H_{0}^{3}(Q)$ and $v \in \stackrel{0}{H}^{3}(Q)$, then by the virtue of $(3.8)\left\langle u, \mathcal{L}^{\prime} v\right\rangle=0$. Consequently, it follows from (4.2) that $v=0$ in $H^{3}(Q)$.

\section{References}

[1] G.C. Barozzi. Su alcuni problemi relative a une equazione lineare a dirivate parziali di tipo composito. Atti Semin. Mat. e Fis. Univ. Modena, 10:11, 19601961.

[2] Yu.M. Berezansky. Expansion in Eigenfunctions of Selfadjoint Operators. Naukova Dumka, Kiev, 1965. (in Russian)

[3] V.I. Burenkov. Sobolev Spaces on Domains. B.G. Teubner, Stuttgart-Leipzig, 1998.

[4] L. Cattabriga. Su alcuni problemi per equazioni differenziali di tipo composito. Rendiconti del Seminario Matematico della Università di Padova, 27:122-143, 1957.

[5] V.V. Dainyak and V.I. Korzyuk. Problem of Dirichlet type for linear differential equation of the third order. Differ. Uravn., 23(5):867-872, 1987. (in Russian)

[6] R.B. Davis. A boundary value problem for third-order linear partial differential equations of composite type. Proc. Amer. Math. Soc., 3:751-756, 1952. Doi:10.1090/S0002-9939-1952-0050788-7.

[7] R.B. Davis. A special case of the normal derivative problem for a third-order composite partial differential equation. Proc. Amer. Math. Soc., 5:720-725, 1954. Doi:10.1090/S0002-9939-1954-0064290-1.

[8] T.D. Dzhuraev. On a certain boundary value problem for an equation of composite type. Dokl. Akad. Nauk Resp. Uzbekistan, 4, 1962. (in Russian)

[9] T.D. Dzhuraev. On a certain boundary value problem for equation $\frac{\partial}{\partial x} \Delta u=0$. In Research on Differential Equations, pp. 46-51, Tashkent, 1963. (in Russian)

[10] G.I. Èskin. Boundary value problem for equation $\frac{\partial}{\partial t} P\left(\frac{\partial}{\partial t}, \frac{\partial}{\partial x}\right) u=0, P\left(\frac{\partial}{\partial t}, \frac{\partial}{\partial x}\right)$ is elliptic operator. Siberian Math. J., 3(6):882-911, 1962. (in Russian)

[11] G.I. Èskin. Boundary-value problems for equations with constant coefficients on the plane. Mat. Sb., 59(101):67-104, 1962. (in Russian)

[12] J. Hadamard. Propriétés d'une équation linéaire aux dérivées partielles du quatrième ordre. Tôhoku Math. J., 37:133-150, 1933.

[13] J. Hadamard. Equations aux derivees partielles. L'enseignement mathematique, 35:5, 1936.

[14] O.A. Konopelko. The boundary value problem for fourth-order equation of composite type. Vestn. BGU. Ser., 1(3):73-79, 2008. (in Russian)

[15] O.A. Konopelko. Weak solutions of boundary value problems in tube domains for a fourth-order equation of composite type. Vestn. BGU. Ser., 1(1):92-98, 2010. (in Russian) 
[16] V.I. Korzyuk. Friedrichs' lemma for mollifiers with variable step. Vestn. BGU. Ser., 1(2):55-71, 1996. (in Russian)

[17] V.I. Korzyuk. Equations of Mathematical Physics. BSU, Minsk, 2010. (in Russian)

[18] V.I. Korzyuk and V.V. Dainyak. On weak solution of problem of Dirichlet type for linear differential equation of the third order. Differ. Uravn., 28(6):10561066, 1992. (in Russian)

[19] V.I. Korzyuk and V.V. Dainyak. On solvability mixed problems for nonstationary equations of the third order. Vestn. BGU. Ser., 1(3):54-60, 2005. (in Russian)

[20] V.I. Korzyuk and O.A. Konopelko. Weak solutions of boundary-value problems in tube domains for the fourth-order equation of composite type. Proceedings of the National Academy of Sciences of Belarus. Physics and Mathematics Series, 3:68-74, 2009. (in Russian)

[21] V.I. Korzyuk and O.A. Konopel'ko. Strong solution of boundary value problems in cylindrical domains for a fourth-order equation of composite type. Differ. Equ., 46(5):690-701, 2010. Doi:10.1134/S0012266110050083.

[22] V.I. Korzyuk, O.A. Konopel'ko and E.S. Cheb. Boundary value problems for the fourth-order equations of hyperbolic and composite types. In Proceedings of the Fifth International Conference on Differential and Functional-Differential Equations (Moscow, August 17-24, 2008). Part 2, volume 36 of CMFD, pp. 87-111, M., 2010. PFUR. Available from Internet: http://mi.mathnet.ru/cmfd158.

[23] V.P. Mikhailov. Partial Differential Equations. Nauka, Moscow, 1976. (in Russian)

[24] O. Sjöstrand. Sur une équation aux dérivées partielles du type composite. Arkiv För Matematik, Astronomi Och Fysik, 25A(21):1-11, 1936.

[25] O. Sjöstrand. Sur une équation aux dérivées partielles du type composite. Note 2. Arkiv För Matematik, Astronomi Och Fysik, 26A(1):1-10, 1939. 